summed over odd $s$, is uniformly convergent in $k$ because of Abel's lemma.

Hence

$(2 / \pi) \sum^{\prime}(r-s)^{-1} \int_{-K}^{K} g(k) \exp \left(i k x_{s}\right) d k=(2 / \pi) \int_{-K}^{K} g(k)\left[\sum^{\prime}(r-s)^{-1} \exp \left(i k x_{s}\right)\right] d k$

$$
=i \int_{-K}^{K} g(k) \exp \left(i k x_{r}\right) d k
$$

in view of (28). However, the transform of $f(x)$, Eq. (29) is given by

$$
T[f(x)]=\pi^{-1} \int_{-\infty}^{\infty}(x-y)^{-1} \int_{-K}^{K} g(k) \exp (i k y) d k d y .
$$

When $x$ is restricted to any finite interval, the order of integration may be interchanged provided that one interprets the singular integral with respect to $y$ as a "principal value". Hence

$$
\begin{aligned}
T[f(x)] & =\pi^{-1} \int_{-K}^{K} g(k) \int_{-\infty}^{\infty}(x-y)^{-1} \exp (i k y) d y d k \\
& =i \int_{-K}^{K} g(k) \exp (i k x) d k
\end{aligned}
$$

which agrees with (30). Therefore the quadrature formula (26) is exact for functions of the form (29) provided that

$$
K \Delta x<\pi
$$

\title{
REFERENCES
}

1. H. Multhopp, Die Berechnung der Auftriebsverteilung von Tragfiügeln, Lufo 15, 153-169 (1938); also available as British RTP Translation 2392.

2. H. Glauert, The elements of aerofoil and airscrew theory, Cambridge University Press, 1930.

\section{NOTE ON THE EXISTENCE AND DETERMINATION OF A VECTOR POTENTIAL*}

\section{By A. F. STEVENSON (Wayne University)}

The problem of finding a solution of the equation

$$
\operatorname{curl} \mathbf{F}=\mathbf{f} \text {, }
$$

where $\mathbf{f}$ is a given vector function of position and $\mathbf{F}$ is to be found, is a classical one, and one would hardly think that there was anything fresh to be said about the matter to-day. In connection with some recent work, however, the writer had occasion to look into the matter somewhat closely, and as a result of this it appeared that the usual treatments $^{1}$ are not satisfactory if $\mathrm{f}$ is only defined in a restricted region. Usually, in

*Received June 23, 1953.

'The following textbooks were consulted: R. Courant, Differential and integral calculus, vol. II (Nordeman, New York, 1944); E. Goursat, Mathematical analysis, vol. I (Ginn, New York, 1904); E. Picard, Traite d'analyse, vol. I (Gauthier-Villars, Paris, 1922); C. E. Weatherburn, Advanced vector analysis (Bell, London, 1928); H. Lass, Vector and tensor analysis (McGraw-Hill, New York, 1950). 
fact, either the region is not mentioned at all, or else it is assumed that it is the whole of space $^{2}$; and the solution ordinarily given does not actually furnish an acceptable solution in some cases.

It is commonly stated that the necessary and sufficient condition for (1) to possess a solution is

$$
\operatorname{div} \mathbf{f}=0,
$$

but we shall see that this may not be sufficient and that other conditions may be necessary.

Taking Cartesian coordinates-as we shall throughout-the solution usually given is obtained by supposing that one of the components of $F$, say $F_{z}$, is zero, leading to

$$
\begin{aligned}
& F_{x}=\int_{c}^{z} f_{\nu} d z+\phi(x, y), \quad F_{\nu}=-\int_{c}^{z} f_{x} d z+\psi(x, y), \\
& F_{z}=0
\end{aligned}
$$

where $c$ is some constant, and, in the integrations with respect to $z, x$ and $y$ are held constant. Taking account of (2), we then find that this is a solution of (1) provided that the functions $\phi, \psi$ satisfy

$$
\frac{\partial \psi}{\partial x}-\frac{\partial \phi}{\partial y}=f_{z}(x, y, c) ;
$$

and this equation can be satisfied, for instance, by taking $\psi=0, \phi$ being then given by a quadrature. More generally, we could take $c$ in (3) to be a function of $x, y$, or even take $c$ to be different functions of $x, y$ in the two integrals; this only leads to a modification of the right-hand side of (4).

The solution (3) implies, however, that the points $(x, y, z)$ and $(x, y, c)$ can be joined by a straight line lying entirely in the region within which $\mathbf{f}$ is defined. This will not be the case for some regions if $c$ is a constant or any continuous function of $x, y$. It can always be achieved by choosing $c$ differently in different portions of the region, but this may cause the solution to be discontinuous. Similar difficulties may arise from the quadratures necessary to solve (4). We can, of course, always find a solution in the form (3) which is valid in a portion of the region concerned, and it may happen that this solution may possess an analytic continuation in the whole region. But clearly cases can arise where (3) does not provide an acceptable solution. We shall give an example below.

We now consider the problem of solving (1) in some region $R$ which, for definiteness, we take to be a simply-connected region bounded by $n+1$ simple closed surfaces with continuous curvature $S_{0}, S_{1}, \cdots, S_{n}$, where $S_{0}$ enclosed $S_{1}, \cdots, S_{n}$, these $n$ surfaces being all exterior to each other. We may have $n=0$, in which case the region is simply the interior of $S_{0}$. We suppose that $\mathrm{f}$ possesses continuous first derivatives in $R$, and require a solution which also possesses continuous first derivatives in $R$.

We shall now prove that the necessary and sufficient conditions for (1) to possess a solution are condition (2), together with the $n+1$ conditions:

$$
\int_{S i} \mathbf{n} \cdot \mathbf{f} d S=0, \quad i=0,1, \cdots, n,
$$

${ }^{2}$ Courant (Ref. 1) considers a region which is the interior of a parallelepiped, thus avoiding the difficulties mentioned here. 
where $\mathbf{n}$ denotes a unit vector along the normal drawn outward from $R$ at any one of the bounding surfaces. It follows from (2) that if any $n$ of the conditions (5) are satisfied, then the remaining condition is also satisfied. If $n=0$, the single condition (5) follows from (2). In general, however, (5) is not a consequence of (2). That conditions (5) are necessary is immediate, since, by Stokes' theorem,

$$
\int_{S i} \mathbf{n} \cdot \operatorname{curl} \mathbf{F} d S=0, \quad i=0,1, \cdots, n .
$$

We shall show that (2) and (5) are sufficient conditions by constructing an explicit solution in terms of the solutions of certain Neumann problems, using for this purpose a generalization of a method used in electromagnetic theory, where, however, it is always supposed that $R$ is the whole of space.

We first extend the definition of $\mathrm{f}$ throughout space. Inside $S_{i}(i=1, \cdots, n)$ we extend it by requiring that (2) be satisfied and that $n \cdot f$ be continuous on $S_{i}$. This can be accomplished by putting, inside $S_{i}$,

$$
\mathbf{f}_{i}=\nabla V_{i}
$$

where $V_{i}$ is a harmonic function which satisfies the boundary condition

$$
\mathbf{n} \cdot \nabla V_{i}=\mathbf{n} \cdot \mathbf{f} \quad \text { on } \quad S_{i} .
$$

This Neumann problem possesses a solution on account of (5). We extend $f$ outside $S_{0}$ in the same manner, and require in addition $f \rightarrow 0$ at infinity. This can be accomplished by solving an exterior Neumann problem; and we note from (5) that the extended $f$ will vanish at infinity to an order $1 / r^{3}$ at least, since an exterior harmonic function which is such that the integral of its normal derivative over a closed surface vanishes, must vanish at infinity to the order $1 / r^{2}$ at least.

We now assert that a solution of (1), for all interior points of $R$, is given by

$$
\mathbf{F}=\operatorname{curl} \mathbf{A}, \quad \mathbf{A}=(1 / 4 \pi) \int \mathbf{f} / r d \tau
$$

where $r$ denotes the distance from a point in the domain of integration to the point at which $\mathbf{A}$ is estimated, the integral being taken throughout space. In the first place, the integral is convergent on account of the behavior of $\mathbf{f}$ at infinity. Further, $\operatorname{div} \mathbf{A}=0$. For the contributions to $\operatorname{div} \mathbf{A}$ from the various regions into which space is divided can, in the usual way, on taking account of (2), be expressed as integrals over the bounding surfaces. The integrals over the surfaces $S_{0}, \cdots, S_{n}$ cancel on account of the boundary conditions imposed on the extended $f$. There remains an integral over the sphere at infinity arising from the region exterior to $S_{0}$. But this also vanishes on account of the behavior of $\mathbf{f}$ at infinity. Hence $\operatorname{div} \mathbf{A}=0$, and therefore

$$
\operatorname{curl} \mathbf{F}=\operatorname{curl} \operatorname{curl} \mathbf{A}=-\nabla^{2} \mathbf{A}=\mathbf{f},
$$

as required. Also since $\mathrm{f}$ possesses continuous first derivatives in $R$, the same is true of $\mathbf{F}$. If $R$ is the unbounded region exterior to $S_{1}, \cdots, S_{n}$, and if $\mathbf{f}$ does not vanish at infinity to the required order, we first surround $S_{1}, \cdots, S_{n}$ by a surface $S_{0}$ and proceed as before. We then obtain a solution valid in the portion of $R$ interior to $S_{0}$, where $S_{0}$ can be taken arbitrarily large. To our particular solution we can, of course, add the gradient of an arbitrary scalar function. 
In the usual physical applications, such as to the magnetic vector potential, the conditions (5) are satisfied as well as (2), and a solution exists. Occasionally, however, the roles of the electric and magnetic fields are interchanged, and the electric field in a charge-free region is expressed as the curl of a vector potential. The above discussion shows that this is not possible in, for instance, the region exterior to a closed surface which encloses a net charge.

Two simple examples, (A) and (B), will illustrate the above considerations, the region $R$ being taken in each case to be that exterior to a sphere of radius $a$ whose center is the origin:

$$
\mathbf{f}=\nabla(1 / r), \quad r=\left(x^{2}+y^{2}+z^{2}\right)^{1 / 2} .
$$

Condition (2) is satisfied, but (5) is not. Therefore there exists no solution. (There will, of course, exist solutions in other regions).

$$
\mathbf{f}=\nabla\left[\left(x^{2}-y^{2}\right) / r^{5}\right] .
$$

Conditions (2) and (5) are both satisfied. If we try to use the solution (3), the simplest choice for $c$ is $c= \pm \infty$, in which case (4) is satisfied by $\phi=\psi=0$. Taking $c=+\infty$, say, (3) gives

$$
\begin{gathered}
F_{x}=\frac{\partial g}{\partial y}, \quad F_{y}=-\frac{\partial g}{\partial x}, \quad F_{z}=0, \\
g=\frac{x^{2}-y^{2}}{\left(x^{2}+y^{2}\right)^{2}}\left(\frac{z}{r}-\frac{1}{3} \frac{z^{3}}{r^{3}}-\frac{2}{3}\right),
\end{gathered}
$$

provided at least one of $x, y$ does not vanish; while if $x=y=0, z \geq a$, we can use the limiting values of the derivatives of $g$ as $x, y \rightarrow 0$. We thus obtain a solution (with continuous derivatives) valid in the portion of $R$ for which $z \geq 0$. If, however, we extend this solution into the portion of $R$ for which $z<0$, it becomes singular on the line $x=y=0, z \leq-a$, since the function $g$ in (7), and its derivatives, do not possess limits as $x, y \rightarrow 0$ for $z<0$. It is readily seen that any other choice of the limits $c$ in (3) (for instance, taking $c=+\infty$ for $z \geq 0$ and $c=-\infty$ for $z<0$ ) either gives a solution which is singular on the positive or negative $z$-axis, or else one which is discontinuous in $R$. Permuting $x, y, z$ in (3) runs into similar difficulties. Thus there exists no solution of the form (3) in which one of the components of $\mathbf{F}$ vanishes.

We can, however, easily obtain a solution in the form (6) by solving the required Neumann problem for the sphere. After some simplification we find:

$$
F_{z}=y z / r^{5}, \quad F_{y}=z x / r^{5}, \quad F_{z}=-2 x y / r^{5} .
$$

This solution is independent of $a$ and is valid everywhere except at the origin. In this particular case, we can obtain the solution more simply by writing

$$
\mathbf{f}=\frac{1}{3} \nabla\left[\frac{\partial^{2}}{\partial x^{2}}\left(\frac{1}{r}\right)-\frac{\partial^{2}}{\partial y^{2}}\left(\frac{1}{r}\right)\right],
$$

and treating the two terms of $f$ separately: for the first term we use a solution of the form (3) with $F_{x}=0$, and for the second term one of the same form with $F_{y}=0$. But this is not, of course, the same as using (3) directly; and in more complicated cases it will be 
difficult, and probably not possible, to express $\mathbf{f}$ as the sum of a finite number of terms for each of which we can use (3).

\section{STRESS FUNCTIONS OF MAXWELL AND MORERA*}

\section{Br WILHELM ORNSTEIN (Newark College of Engineering)}

Summary. A systematic process is devised for the derivation of the stress functions of Maxwell and Morera by the application of the theorem that a vector whose divergence vanishes is solenoidal. Symmetric and anti-symmetric matrices are established, and the elements of these matrices represent the stress functions of Maxwell and Morera respectively.

Introduction. The procedure used by $\mathrm{Maxwell}^{1}$ to derive three stress functions representing six stress components at any point of an isotropic body is similar to that which Morera ${ }^{2}$ subsequently applied to the establishment of the corresponding stress functions. This procedure described also by Love ${ }^{3}$ consisted in the choice of three stress components; then the substitution of these components into the equilibrium equation led to the remaining three components necessary to satisfy the equations of equilibrium. Later it was discovered by Sir Richard Southwell ${ }^{4}$ that Saint Venant's and Beltrami's compatibility equations follow from Castigliano's principle when the strain energy is expressed in terms of Maxwell's and of Morera's functions.

Derivations. Neglecting the body forces, the equation of equilibrium is

$$
\operatorname{div} T=0 \text {, }
$$

where $T$ is the stress tensor. The three equations of equilibrium are obtained by cyclic interchange of $x, y, z$ in the equation

$$
\frac{\partial X x}{\partial x}+\frac{\partial X y}{\partial y}+\frac{\partial X z}{\partial z}=0 \text {. }
$$

The equations of equilibrium (2) can be written as

$$
\operatorname{div} A=0, \quad \operatorname{div} B=0, \quad \operatorname{div} C=0
$$

where, because of the theorem that a vector whose divergence vanishes is a solenoidal vector, the following relations exist:

$$
A=\operatorname{curl} F, \quad B=\operatorname{curl} G, \quad C=\operatorname{curl} H .
$$

Consequently, the stress components may be written as follows:

$$
X x=\frac{\partial F_{3}}{\partial y}-\frac{\partial F_{2}}{\partial z}, \quad X y=\frac{\partial F_{1}}{\partial z}-\frac{\partial F_{3}}{\partial x}, \quad X z=\frac{\partial F_{2}}{\partial x}-\frac{\partial F_{1}}{\partial y} ;
$$

*Received September 2, 1953.

${ }^{1}$ C. Maxwell, Edinburgh Royal Soc. Trans. 26 (1870).

${ }^{2}$ G. Morera, Roma, Acc. Lincei Rend. (5) 1 (1892).

${ }^{3}$ A. E. H. Love, $A$ treatise on the mathematical theory of elasticity, Fourth Edition, 1927, p. 88.

${ }^{4}$ R. V. Southwell, Proc. Roy. Soc. (A) 154 (1925). R. V. Southwell, Timoshenko Anniversary Volume, pp. 211-216. 